\title{
Opening angles and shapes of parsec-scale AGN jets
}

\author{
Alexander B. Pushkarev*† \\ Pulkovo Observatory, Pulkovskoe Chaussee 65/1, St. Petersburg 196140, Russia \\ Crimean Astrophysical Observatory, Nauchny 298409, Crimea, Russia \\ Max-Planck-Institut für Radioastronomie, Auf dem Hügel 69, 53123 Bonn, Germany \\ E-mail: pushkarev.alexander@gmail.com
}

\section{Matthew L. Lister}

Department of Physics, Purdue University, 525 Northwestern Avenue, West Lafayette, IN 47907, USA

\section{Yuri Y. Kovalev}

Astro Space Center of Lebedev Physical Institute, Profsoyuznaya 84/32, Moscow 117997, Russia Max-Planck-Institut für Radioastronomie, Auf dem Hügel 69, 53123 Bonn, Germany

\section{Tuomas Savolainen}

Max-Planck-Institut für Radioastronomie, Auf dem Hügel 69, 53123 Bonn, Germany Aalto University Metsähovi Radio Observatory, Metsähovintie 114, 02540 Kylmälä, Finland

We used $15 \mathrm{GHz}$ VLBA observations of 363 sources having at least 5 epochs within a time interval 1995-2013 from the MOJAVE program and/or its predecessor, the $2 \mathrm{~cm}$ VLBA Survey. For each source we produced a corresponding stacked image averaging all available epochs for a better reconstruction of the cross section of the flow. We have analyzed jet profiles transverse to the local jet ridge line and derived both apparent and intrinsic opening angles of the parsecscale outflows. The sources detected by the Fermi Large Area Telescope (LAT) during the first 24 months of operation show wider apparent jet opening angle and smaller viewing angles on a very high level of significance supporting our early findings. Analyzing transverse shapes of the outflows we found that most sources have conical jet geometry at parsec scales, though there are also sources that exhibit active jet collimation.

12th European VLBI Network Symposium and Users Meeting,

7-10 October, 2014

Cagliari, Italy

\footnotetext{
*Speaker.

Acknowledgments. This research has made use of data from the MOJAVE database that is maintained by the MOJAVE team. The MOJAVE project was supported under NASA-Fermi grants NNX12A087G and 11-Fermi11-0019. The National Radio Astronomy Observatory is a facility of the National Science Foundation operated under cooperative agreement by Associated Universities, Inc. This study was supported in part by the Russian Foundation for Basic Research grant 13-02-12103.
} 

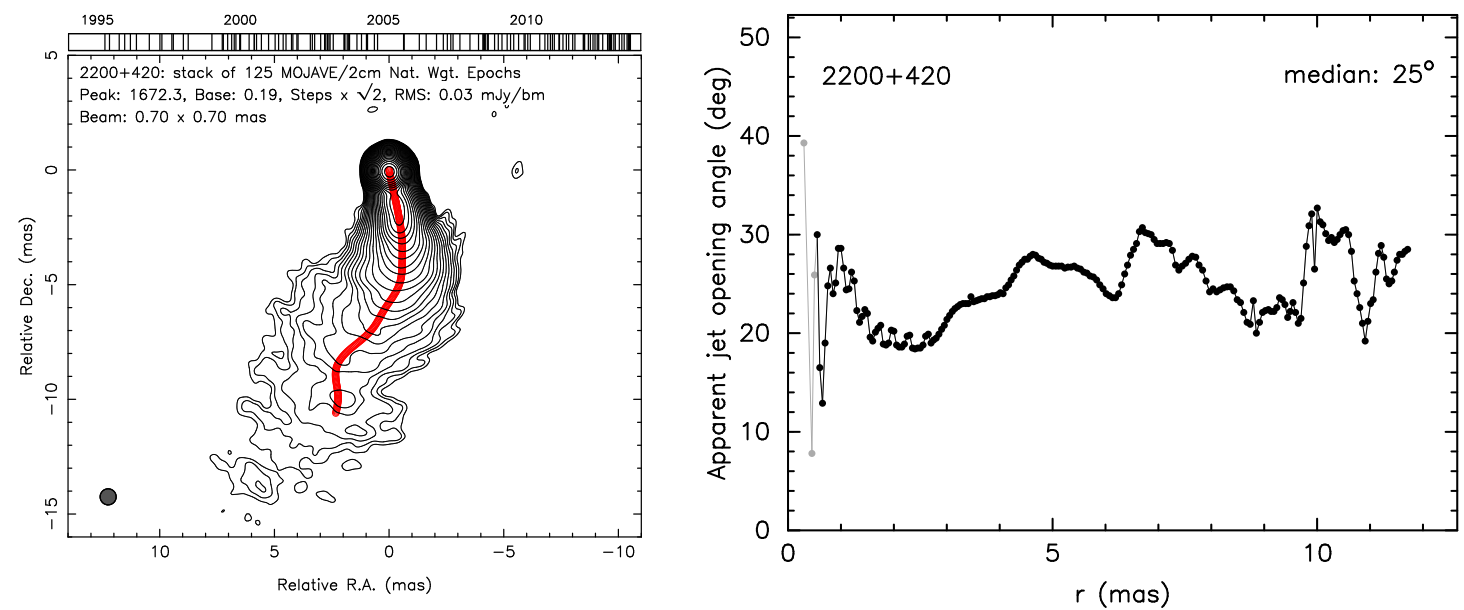

Figure 1: Left: MOJAVE image of BL Lac at $15 \mathrm{GHz}$ after stacking 125 epochs covering a time range of about 20 years. The total intensity ridge line is shown by red. Time evolution of the ridge line in BL Lac is discussed in [2]. Right: Apparent opening angle of the jet along its ridge line.

\section{Apparent opening angles}

Recently we found that nearly all of the 60 heavily observed jets display significant changes of the innermost jet position angle with time [1] suggesting that the superluminal AGN jet features seen in a single-epoch images occupy only a portion of the entire jet cross section. Thus, to better reconstruct the jet cross section of each source observed within the MOJAVE/2cm VLBA Survey we produced a corresponding stacked image using all available epochs for a given source at $15 \mathrm{GHz}$, comprising a sample of 363 AGN jets having at least 5 epochs and a clear VLBI core position. The opening angle of the jet was calculated as the median value of $\alpha_{\text {app }}=2 \arctan \left[0.5\left(D^{2}-\right.\right.$ $\left.b_{\varphi}^{2}\right)^{1 / 2} / r$, where $D$ is the FWHM of a Gaussian fitted to the transverse jet brightness profile, $r$ is the distance to the VLBI core along the jet ridge line, $b_{\varphi}$ is the beam size along the position angle $\varphi$ of the jet-cut. In Fig. 1 we show the $15 \mathrm{GHz}$ stacked image of BL Lac as an example together with opening angle of the jet as a function of angular distance to the core along the ridge line.

The distribution of the derived $\alpha_{\text {app }}$ values is shown in Fig. 2 (top). LAT-detected [3] sources (Fig. 2, bottom) have statistically wider apparent jet opening angles compared to those of non-LATdetected (Fig. 2, middle) on a very high level of significance supporting our early findings.

\section{Intrinsic opening angles and viewing angles}

We have derived the values of the viewing angle $\theta$ and the bulk Lorentz factor $\Gamma$ using jet speeds from the MOJAVE kinematic analysis [1] and variability Doppler factor from the Metsähovi AGN monitoring program [4]. The overlap of the MOJAVE and Metsähovi programs comprises 56 sources. The intrinsic opening angles calculated for the 56 sources using a relation $\tan \left(\alpha_{\text {int }} / 2\right)=$ $\tan \left(\alpha_{\text {app }} / 2\right) \sin \theta$ have a median of 1.3 and show inverse dependence on Lorentz factor (Fig. 3, left), as predicted by hydrodynamical [5] and magnetic acceleration models [6] of relativistic jets.

A K-S test indicates no significant difference $(p=0.43)$ between the samples of LAT-detected and non-LAT-detected sources, suggesting that the established statistical difference in apparent 


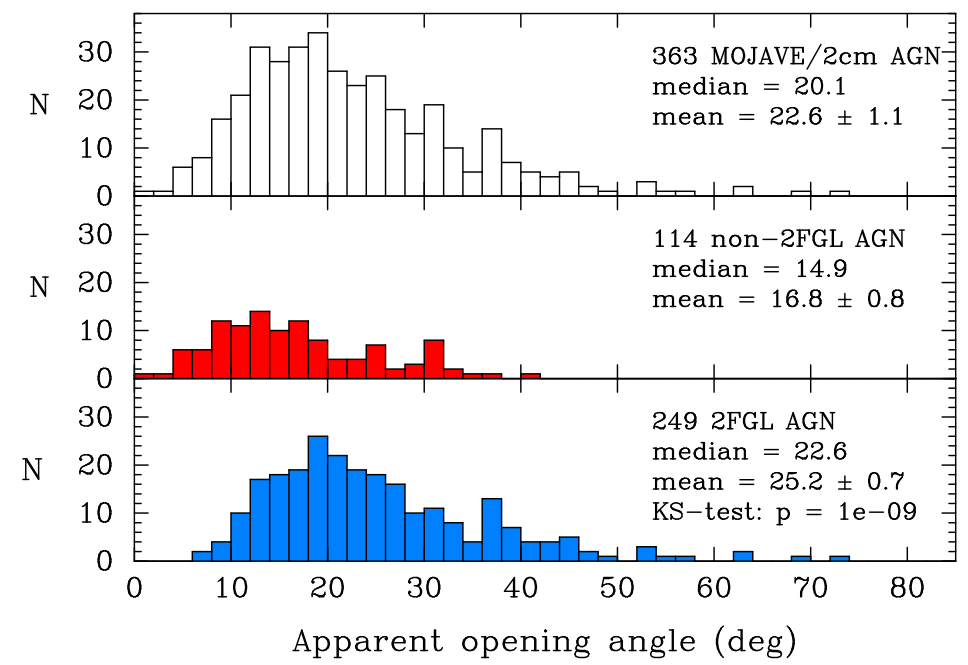

Figure 2: Distributions of the apparent opening angle from jet-cut analysis for 363 MOJAVE AGN (top panel), comprising 114 non-LAT-detected (middle panel) and 249 LAT-detected (bottom panel) sources after 24 months of scientific operation.
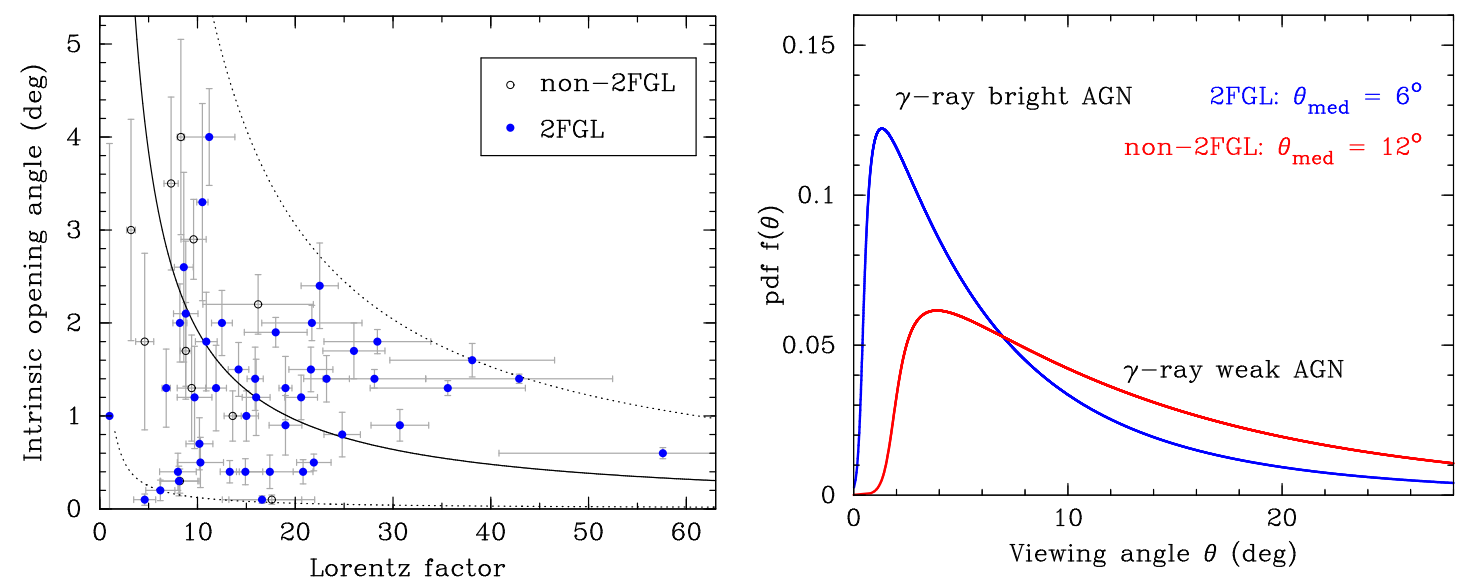

Figure 3: Left: Intrinsic opening angle vs. Lorentz factor for 56 jets. The solid line shows the median curve fit with the assumed relation $\alpha_{\text {int }}=\rho / \Gamma$, where $\rho$ is a constant (here $\rho=0.33$ rad). Right: Probability density function of viewing angle as derived from the apparent angle and Lorentz factor distributions of $\gamma$-ray bright (blue curve) and $\gamma$-ray weak (red curve) AGN.

opening angles is the result of projection effects, i.e., the $\gamma$-ray bright jets are viewed at preferentially smaller angles. Indeed, using Monte-Carlo simulation together with the Generalized Lambda Distribution technique we have derived probability density functions of viewing angle for the LATdetected and non-LAT-detected sources (Fig. 3, right), showing that jets of the $\gamma$-ray bright AGN tend to have smaller angles to the line of sight comparing to those of $\gamma$-ray weak AGN, with median values $6^{\circ}$ and $12^{\circ}$, respectively.

\section{Jet shapes}

To study shapes of the outflows, we analyzed dependence between jet widths $d=\left(D^{2}-b_{\varphi}^{2}\right)^{1 / 2}$ derived from the profiles transverse to the local jet direction and angular separation $r$ measured 

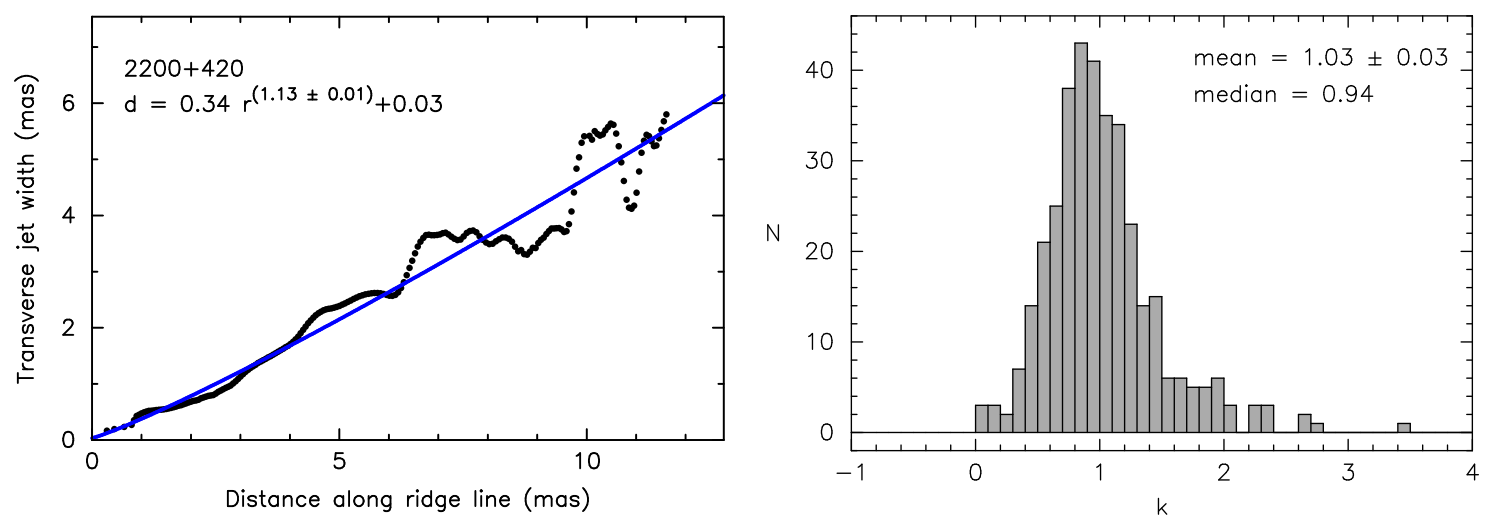

Figure 4: Left: Jet width $d$ vs distance $r$ for BL Lac. The blue line is the best fit of an assumed power-law dependence $d \propto r^{k}$. Right: Distribution of the power-law index $k$, with a peak at $k \sim 1$ implying that a jet shape is close to conical for most parsec-scale AGN outflows.

along the reconstructed total intensity ridge line. We assumed a power-law dependence $d \propto r^{k}$ and searched for the best fit space parameters using $\chi^{2}$ minimization (Fig. 4, left).

The distribution of the derived power-law index $k$ presented in Fig. 4, (right) peaks at values close to 1 , suggesting that parsec-scale AGN jets typically manifest a shape close to conical. At the same time, a number of sources show $k$ values significantly smaller than 1 , indicating that the jets undergo collimation on scales probed by our observations (e.g., $k=0.47 \pm 0.01$ for M 87). BL Lacs and quasars have similar $k$-distributions, with the means of $1.13 \pm 0.06$ and $1.01 \pm 0.04$, respectively, whereas galaxies show on average smaller $k=0.83 \pm 0.12$, most probably because these objects are systematically closer, and their jets are oriented at larger angles to the line of sight, allowing us to probe the jet regions at shorter linear separations from the central engine where the outflows become organized more effectively [7].

\section{References}

[1] M.L. Lister, M.F. Aller, H.D. Aller, et al., MOJAVE. X. Parsec-scale Jet Orientation Variations and Superluminal Motion in Active Galactic Nuclei, ApJ 146, 120 (2013).

[2] M.H. Cohen, D.L. Meier, T.G. Arshakian, et al., Studies of the Jet in BL Lacertae. II. Superluminal Alfvén Waves, arXiv: 1409.3599 (2014).

[3] M. Ackermann, M. Ajello, A. Allafort, et al., The Second Catalog of Active Galactic Nuclei Detected by the Fermi Large Area Telescope, ApJ 743, 171 (2011).

[4] T. Hovatta, E. Valtaoja, M. Tornikoski, A. Lähteenmäki, Doppler factors, Lorentz factors and viewing angles for quasars, BL Lacertae objects and radio galaxies, A\&A 498, 723 (2009).

[5] R.D. Blandford \& A. Königl, Relativistic jets as compact radio sources, ApJ 232, 34 (1979).

[6] S.S. Komissarov, M.V. Barkov, N. Vlahakis, \& A. Königl, Magnetic acceleration of relativistic active galactic nucleus jets, MNRAS 380, 51 (2007).

[7] K. Hada, M. Kino, A. Doi, et al., The Innermost Collimation Structure of the M87 Jet Down to 10 Schwarzschild Radii, ApJ 775, 70 (2013). 